National training surveys (GMC/Scottish training survey) have highlighted the need for an inclusive approach and collaborative culture. The aim of the TLs is to engage trainees, promoting better communication and model a positive environment for learning and quality improvement (QI).

How we did it There are nine TLs across the divisions of Medicine, Surgery, Women's and Children and Support Services. The role of TLs is to engage with trainees within their Divisions by having regular meetings with trainees and feeding back to the medical education team within the health board. By participating in medical education and managerial meetings, TLs have developed management skills and driven quality improvement projects.

The monthly Trainee Forum was set up to raise awareness of the roles and structure of the management team. Guest speakers from the management Divisions have provided trainees with an insight into how the health board functions and provided a platform to raise issues directly with the management team.

Some examples of initiatives include trainee-led QI projects to enhance teaching opportunities include rota restructuring, development of hospital at night for surgery to ensure safe and adequate cover out of hours, involvement of trainees in organisation of morbidity and mortality meetings.

Furthermore, a monthly Trainee Newsletter was set up to signpost trainees on upcoming forums and advertise QI sessions and workshops.

Outcomes The TL initiative is an ongoing project with regular feedback in the form of TL meetings, forums and trainees. As TL's we have shared methodologies that have worked well to create a supportive and nurturing environment.

The initiative has resulted in increased awareness of management and their vital role in the running of the National Health Service. This in turn has led to improved trainee engagement. On a fundamental level, trainees have been able to raise issues and be part of implementing change.

The future Through collaboration between trainees, across Divisions and with management, we will continue to engage trainees, sustain change and build resilience.

\section{G400(P) AN INTEGRATED 'ONE-STOP' MULTI-DISCIPLINARY (MDT) CLINIC FOR CHILDREN AND YOUNG PEOPLE WITH TUBEROUS SCLEROSIS COMPLEX (TSC)}

\footnotetext{
${ }^{1} \mathrm{M}$ Raja, ${ }^{2} \mathrm{~S}$ Philip, ${ }^{2} \mathrm{~S}$ Agrawal, ${ }^{3} \mathrm{G}$ McKerracher, ${ }^{4} \mathrm{~K}$ Rhodehouse, ${ }^{5} \mathrm{~J}$ Tuberville-Greenley, ${ }^{5}$ J Hussain, 'L Kerecuk. 'Nephrology, Birmingham Women's and Children's NHS Foundation Trust, Birmingham, UK; ${ }^{2}$ Neurology, Birmingham Women's and Children's NHS Foundation Trust, Birmingham, UK; ${ }^{3}$ Psychology, Birmingham Women's and Children's NHS Foundation Trust, Birmingham, UK; ${ }^{4}$ Tuberous Sclerosis Association, Tuberous Sclerosis Association, London, UK; ${ }^{5}$ Centre for Rare Disease, Birmingham Women's and Children's NHS Foundation Trust, Birmingham, UK
}

\subsection{6/archdischild-2018-rcpch.389}

TSC is a complex, genetic disorder characterised by growth of benign tumours in various organs leading to epilepsy, renal, cardiac, skin, lung, liver and eye manifestations. Thus, patients affected attend multiple clinic appointments with different specialists. Developmental delay and TSC-associated neuropsychiatric disorders (TANDS) are common but often not addressed. Aims

- To improve the care of children and young people affected by TSC by an integrated multidisciplinary team (MDT) 'onestop' clinic.
- To provide psychological assessment of TANDS.

Method The MDT TSC clinic was launched in April 2016 by coordinating neurology, nephrology, oncology, psychology consultants with the Rhoald Dhal Rare Disease (RD) Specialist nurses and Tuberous Sclerosis Advisor (TSA) on same visit. Psychology input was funded by a Pharma Joint Working Agreement. The RD nurse provides Information Packs and calls families to discuss what their main concerns are, thereby enabling clinicians to address what is relevant to patients.

Results The TSC MDT clinic has:

Improved coordination of specialists and investigations.

Feedback from Patient

Questionnaires:

'this should have been started a long time ago', 'this is a fabulous idea, saves us time', 'having all the specialists together makes so much sense -they can explain in a way that we can understand'.

$55 \%$ of families were not aware of the TSA

98 patients identified with TSC by joining lists kept by each specialty. Average patient age 10.3 years. (range: $1.6-$

21.1 years.). $38 \%$ had previous renal input; $42 \%$ had not had a renal ultrasound. $66 \%$ had neurology input but $33 \%$ had no brain imaging.

The Appointment Burden before TSC clinic was reviewed: showed average appointment number/patient was 5.3/year (range $0.6-11.7 /$ yr.).

$40 \%$ of Patients in TSC clinic required further psychology input 20\% referred for formal CAMHS review.

Patients above age of 16 years. reviewed by the adult TSC transition nurses from adult sister hospital.

Conclusion The MDT TSC clinic has improved coordinated patient care including ensuring imaging is regularly carried out. It has identified high burden of psychological need. Overall, this 'one-stop' clinic system has improved the care the patients and families with TSC.

\section{G401(P) DEVELOPMENT OF A PAEDIATRIC SPECIALIST MULTIDISCIPLINARY DOWN SYNDROME CLINIC}

Z Abdelrahim, A Dooley, A Khan. Community Paediatrics, Southern Health and Social Care Trust, Newry, UK

\subsection{6/archdischild-2018-rcpch.390}

Background Down syndrome (Trisomy 21) is a chromosomal condition with special health care needs and single most common identifiable cause of learning difficulties. Medical conditions such as thyroid dysfunction, structural heart disease, problems with hearing, vision and growth are significantly higher in these patients. Evidence suggests that early screening, diagnosis and medical management of these problems can significant reduce secondary illnesses resulting in improved quality of life. Therefore children with Down syndrome requires long-term follow-up in dedicated specialist clinics.This Quality Improvement (QI) project was undertaken as medical care for most Down syndrome children were not met in line with national standards.

Aims To develop a patient centred high quality service in a specialist multidisciplinary Down syndrome clinic to ensure standardised, evidence based medical care in line with national standards. 
Methods A retrospective Audit of current services for children with Down syndrome was completed which identified number of deficiencies within existing services. Recommendations included; establishing a specialist multidisciplinary Down Syndrome Clinic and developing local care pathway for improved documentation and communication. Quality Improvement tools including; Process Flow Analysis, Fishbone diagram, Purpose to practice, and Parent feedback questionnaire were used during this project.

Results and conclusion A specialist Multidisciplinary Down Syndrome clinic was established with first clinic in January 2017. Pilot of once a month clinic with PDSA cycle for 6 months. A local care pathway was developed including core clinic members, clinic frequency, templates, referral criterion and MDT communication framework. Children and young people with Down syndrome are involved in service development. Promoted joined up working in partnership with patients and families

\section{REFERENCES}

1. Guidance for Essential Medical Surveillance for Children with Down syndrome. www.dsmig.org.uk

2. Paediatric Service Specification. Services for Children \& Young people with Down syndrome. RCPCH.

\section{G402(P) THE COMPLETION OF ADVANCE CARE PLANS}

${ }^{1} \mathrm{~K}$ Renton, ${ }^{2} \mathrm{~A}$ Mayer, ${ }^{1,2,3} \mathrm{~F}$ McElligott. 'Paediatric Palliative Care, Leeds General Infirmary/ Martin House Hospice, Leeds, UKi ${ }^{2}$ Paediatric Palliative Care, Sheffield Children's Hospital, Sheffield, UK; ${ }^{3}$ Paediatric Palliative Care, Bluebell Wood Children's Hospice, Sheffield, UK

10.1136/archdischild-2018-rcpch.391

\section{Aims}

- To establish the availability and completion of Advance Care Plans (ACP) in paediatric palliative hospice patients.

- To review changes to this in light of new guidance and following recommendations from a previous audit.

ACPs are a valuable resource for patients, their families and healthcare professionals. Between the first and second audits there were two significant implementations. The National Institute for Health and Care Excellence (NICE) published guidelines about end of life care in children and young people in 2016. One of their quality statements is that all children with a life-limiting condition should be involved in developing an ACP. The second implementation was the adoption of the Children and Young Person's Advance Care Plan (CYPACP) by the local palliative network.

Methods All patients under the care of the palliative medical team were identified. Patients referred within the previous two months were excluded. Notes of all patients were reviewed and computer records accessed. The presence of an ACP was noted and, if available, was reviewed against set standards based on national recommendations. This included items such as patient identifiers, documented discussions about place of care, signature completion, and others.

The first audit was done in December $2016(n=41)$, with a repeat audit (to complete the cycle) in September 2017 $(n=41)$ following institution of recommendations from the first audit. These recommendations included implementation of a teaching programme about using the new proforma (CYPACP).

Results
- In 2016, 80\% of patients had ACPs. This improved in 2017 to $88 \%$.

- In 2017, 25\% were on CYPACP proforma compared to $6 \%$ in 2016.

- Patient identifiers were consistently well completed in both audits.

- Many ACPs were not signed (62\% in 2016, 78\% in 2017) which poses risk and challenges for nurses expected to follow documented recommendations.

- Discussion regarding place of care/death was completed in $100 \%$ of cases in 2017, compared to $79 \%$ in 2016.

Conclusion The process of writing ACPs is time intensive and transfer to new documentation is a significant undertaking. However, the introduction of the CYPACP has led to more comprehensively completed ACPs, particularly around place of care/death.

\section{G403(P) SURVEY OF STAKEHOLDERS OF A PAEDIATRIC ANAESTHETIC ROOM}

${ }^{1} \mathrm{~S}$ Abid, ' $\mathrm{N}$ Greenshields, ${ }^{2} \mathrm{~J}$ Lowe. ${ }^{1}$ Imperial School of Anaesthesia, London Deanery, London, UK; ${ }^{2}$ St Mary's Hospital, Imperial College Healthcare NHS Trust, London, UK

10.1136/archdischild-2018-rcpch.392

Aims To quantify and evaluate stakeholder satisfaction of our paediatric anaesthetic room (PAR), which at present contains limited child-friendly interventions.

Methods We conducted a survey using anonymous paper questionnaires offered to patients, parents and staff over two weeks (29th March - 12th April 2017). Questionnaires were dispensed by nurses and anaesthetic trainees in the paediatric ward and PAR, and collected anonymously via collection boxes.

Inclusion criteria: paediatric patients undergoing elective surgery, parents accompanying patient to PAR; all staff using the PAR (paediatric consultant anaesthetists and operating department practitioners; paediatric nurses and play-therapists accompanying patients)

Exclusion criteria: stakeholder refusal; not fluent in English. Our survey investigated stakeholder satisfaction of the PAR and elicited particular feedback on lighting, colour, toys, and multimedia (tablet/television/music). At the time of the survey, there was no functioning television, no available tablets or music, and soft toys were not routinely used (none were used during the survey period).

Results We collected 58 responses: 9 patients, 20 parents, 29 staff.

Patients: In the PAR, 4/9 (45\%) felt indifferent, 3/9 (33\%) felt negatively and only $2 / 9$ (22\%) felt positively. All respondents wanted a change in colour and décor, and all wanted to play with toys. Almost all $(8 / 9,89 \%)$ wanted some form of multimedia.

Parents: $13 / 20$ parents (65\%) felt the general environment, colour and décor were not child-friendly. The same proportion felt available toys and multimedia were insufficient. 9/20 (45\%) felt lighting was poor.

Staff: $26 / 29$ (90\%) felt the PAR was not child-friendly and the colour/décor was poor. $28 / 29$ (98\%) felt there was insufficient multimedia, 21/29 (72\%) felt selection of toys was inadequate and $16 / 17$ (94\%) felt they preferred a previous PAR they had worked at. 